\title{
Numerical investigation of the radial quadrupole and scissors modes in trapped gases
}

\author{
Lei $\mathrm{Wu}$ and Yonghao Zhang \\ Department of Mechanical \& Aerospace Engineering, \\ University of Strathclyde, Glasgow, G1 1XJ, UK
}

\begin{abstract}
The analytical expressions for the frequency and damping of the radial quadrupole and scissors modes, as obtained from the method of moments, are limited to the harmonic potential. In addition, the analytical results may not be sufficiently accurate as an average relaxation time is used and the high-order moments are ignored. Here, we propose to numerically solve the Boltzmann model equation in the hydrodynamic, transition, and collisionless regimes to study mode frequecy and damping. When the gas is trapped by the harmonic potential, we find that the analytical expressions underestimate the damping in the transition regime. In addition, we demonstrate that the numerical simulations are able to provide reasonable predictions for the collective oscillations in the Gaussian potentials.
\end{abstract}

PACS numbers: 67.85.Lm, 05.20.Dd, 51.10.+y

Introduction. The realization of quantum degeneracy in the ultracold atomic gases has attracted intensive research effort to understand the interacting quantum systems $[1,2]$. The experimental controllability of the interactions, energy, and spin population makes these systems ideal to study the BEC-BCS crossover, which is ubiquitous in high-temperature superconductivity, neutron stars, nuclear matter, and quark-gluon plasma [3]. And the study of collective excitations is important for probing the properties of strongly correlated systems, revealing the underlying mechanics of BEC-BCS crossover.

The dynamics of the dilute quantum gas in the normal phase can be described by the semi-classical Boltzmann equation [4]. The analytical expressions for the mode frequency and damping can be obtained by applying the method of moments to the linearized Boltzmann equation [5-9]. However, the analytical method of moments may not provide accurate predictions, e.g. for the two-component Fermi gases in the transition regime $[9,10]$, which is caused by i) the spatially-dependent relaxation time is replaced by the spatially-average one and ii) only low orders of moments are included in the analytical method, which may not be adequate for capturing the important features of the collective oscillations. For example, one needs to consider high-order terms for the cloud surface deformation at large radii in the quadrupole mode [10]. The other major drawback of the analytical method is that it is only limited to the harmonic potentials, while in experiments anharmonic effects emerge at high temperatures where the external potential has a Gaussian profile $[9,11]$. Therefore, it is necessary to solve the Boltzmann equation numerically to get the mode frequency and damping, which is the aim of this work.

Here we put forward a deterministic method to numerically solve the Boltzmann model equation in the hydrodynamic, transition, and collisionless regimes. For simplicity, we consider two-dimensional gases trapped in the harmonic potential

$$
V(x, y)=\frac{m}{2}\left(\omega_{x}^{2} x^{2}+\omega_{y}^{2} y^{2}\right)
$$

and the Gaussian potential

$$
V(x, y)=V_{0}\left[1-\exp \left(-\frac{x^{2}}{a^{2}}-\frac{y^{2}}{b^{2}}\right)\right],
$$

with the atom mass $m$, spatial coordinates $x$ and $y$, trap frequencies $\omega_{x}$ and $\omega_{y}$, trap depth $V_{0}$, and trap widthes $a$ and $b$. Experimentally, this corresponds to the gas confined in elongated traps so that one can focus on radial oscillations, neglecting the axial motion $[11,12]$. We will compare the predicted mode frequency and damping with the analytical and experimental data $[6,9,13]$.

Model equation and numerical scheme. We study the dilute quantum gases well above the degeneracy temperature, where the gases are statistically classical. Instead of the Boltzmann equation, we begin with the kinetic model equation based on the relaxation-time approximation:

$$
\frac{\partial f}{\partial t}+v_{x} \frac{\partial f}{\partial x}+v_{y} \frac{\partial f}{\partial y}+a_{x} \frac{\partial f}{\partial v_{x}}+a_{y} \frac{\partial f}{\partial v_{y}}=\frac{f_{l e}-f}{\tau(x, y)},
$$

where $f\left(x, y, v_{x}, v_{y} ; t\right)$ is the reduced distribution function which neglects the axial motion, $v_{x}$ and $v_{y}$ are velocity components, $\left(a_{x}, a_{y}\right)=-(\partial / \partial x+\partial / \partial y) V / m$ are the accelerations, $\tau(x, y)$ is the local relaxation time, and $f_{l e}$ is the local equilibrium distribution function

$$
f_{l e}=\frac{m n}{2 \pi k_{B} T} \exp \left[-m \frac{\left(v_{x}-u_{x}\right)^{2}+\left(v_{y}-u_{y}\right)^{2}}{2 k_{B} T}\right],
$$

which is defined in terms of the local particle density $n(x, y)$, local temperature $T(x, y)$, local macroscopic velocities $u_{x}(x, y)$ and $u_{y}(x, y)$, and the Boltzmann constant $k_{B}$. When the system is in global equilibrium, $n=n_{0} \exp \left[-V(x, y) / k_{B} T_{0}\right]$ with $n_{0}$ the particle density at the trap center and $T_{0}$ the global equilibrium temperature.

The shear viscosity plays a dominant role in the collective oscillations; the cloud remains nearly isothermal and the experiment is not sensitive to the thermal conductivity $[14,15]$. Therefore, the local relaxation time can be determined by equating the shear viscosity with that derived from the kinetic model equation 
(3), yielding $\tau=\eta / n k_{B} T$. When the vacuum expression for the cross section is used, we have $\tau(x, y)=$ $15 \sqrt{m \pi / k_{B} T} / 16 \sigma n \int_{0}^{\infty} d \xi \xi^{7} e^{-\xi^{2}}\left(1+\xi^{2} T / T_{a}\right)^{-1}$, where $\sigma$ is the total energy-independent cross section $\left(4 \pi a^{2}\right.$ and $8 \pi a^{2}$ for the Fermi and the Bose gases, respectively) and $T_{a}=\hbar^{2} / m k_{B} a^{2}$, with $a$ the s-wave scattering length and $\hbar$ the Planck constant. Two limiting cases will be considered. When $a$ is small, the scattering cross section is energy-independent, and atoms behave like hard spheres. The local relaxation time is therefore given by $[16,17]$

$$
\tau(x, y)=\frac{5}{16 \sigma n(x, y)} \sqrt{\frac{m \pi}{k_{B} T(x, y)}} .
$$

On the contrary, in the unitarity limit where $a \rightarrow \infty$, we have, for example, for the Fermi gases

$$
\tau(x, y)=\frac{15 m^{3 / 2}}{64 \hbar^{2} n(x, y)} \sqrt{\frac{k_{B} T(x, y)}{\pi}} .
$$

We adopt the concept of asymptotic preserving to solve the model equation (3) numerically [18]. The terms at the left side are treated explicitly, while the collision term at the right side is treated implicitly to overcome its stiffness in the hydrodynamic regime (the stiff means that, since the relaxation time $\tau(x, y)$ is very small, numerical errors can be greatly amplified), resulting

$$
\frac{f^{j+1}-f^{j}}{\Delta t}+\operatorname{Tr}\left[f^{j}\right]=\frac{1}{\tau^{j+1}(x, y)}\left(f_{l e}^{j+1}-f^{j+1}\right),
$$

where $\Delta t$ is the time step, variables with superscript $j$ denote the values of these variables at the $j$-th time step, and $\operatorname{Tr}\left[f^{j}\right]$ represents the spatial and velocity discretization of the last four terms at the left side of Eq. (3). If the spatial and velocity ranges are wide enough such that $f$ is zero at the boundaries, $\operatorname{Tr}\left[f^{j}\right]$ can be handled by the fast Fourier transformation. By using the conservative properties of the collision term, the nonlinear implicit equation (7) can be solved explicitly. That is, given $f^{j}$, one can get $n^{j+1}, u_{x}^{j+1}, u_{y}^{j+1}$, and $T^{j+1}$ from the following equations: $n^{j+1}=\iint\left(f^{j}-\Delta t T\left[f^{j}\right]\right) d v_{x} d v_{y}, u_{x}^{j+1}=$ $\iint v_{x}\left(f^{j}-\Delta t T\left[f^{j}\right]\right) d v_{x} d v_{y} / n^{j+1}, u_{y}^{j+1}=\iint v_{y}\left(f^{j}-\right.$ $\left.\Delta t T\left[f^{j}\right]\right) d v_{x} d v_{y} / n^{j+1}$, and $T^{j+1}=\iint m\left(v_{x}^{2}+v_{y}^{2}\right)\left(f^{j}-\right.$ $\left.\Delta t T\left[f^{j}\right]\right) d v_{x} d v_{y} / 2 n k_{B}-m\left(u_{x}^{2}+u_{y}^{2}\right) / 2 k_{B}$, where the integration can be carried out by direct discrete sum or by the Simpson's rule. The above four macroscopic quantities at the $(j+1)$-th time step determine $\tau^{j+1}$ and $f_{l e}^{j+1}$. Therefore, $f^{j+1}$ can be solved explicitly.

The virtual of the asymptotic preserving scheme is that it can capture gas dynamics in the hydrodynamic limit even if the small scale determined by the relaxation time is not numerically resolved. The computational accuracy in the hydrodynamic regime is guaranteed by the fact that, using the Chapman-Enskog expansion [19], Eq. (7) reduces to the Euler equations when $\tau$ is very small. Therefore, the computation of a hydrodynamic flow can be as fast and accurate as that of the transitional and
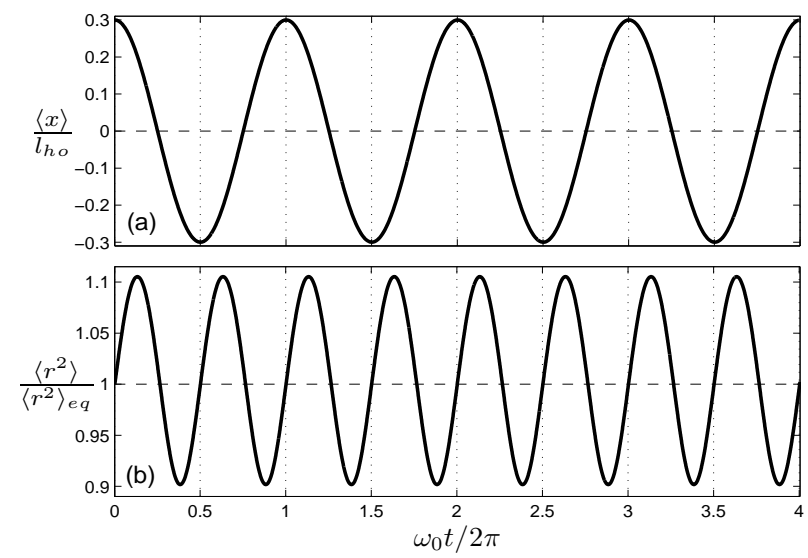

FIG. 1: Numerical simulation of (a) the sloshing mode and (b) the breathing mode. The initial distribution function is $f=\exp \left\{-\left[\omega_{0}^{2}\left(x-0.3 l_{h o}\right)^{2}+\omega_{0}^{2} y^{2}+v_{x}^{2}+v_{y}^{2}\right] / 2\right\} / 2 \pi$ for the sloshing mode and $f=\exp \left\{-\left[\omega_{0}^{2}\left(x^{2}+y^{2}\right)+\left(v_{x}-0.8 x\right)^{2}+\left(v_{y}-\right.\right.\right.$ $\left.\left.0.8 y)^{2}\right] / 2\right\} / 2 \pi$ for the breathing mode. In both simulations, $m=k_{B}=T_{0}=1, \omega_{0}=\sigma=4$, so that the characteristic length $l_{h o}$ is 0.25 and the system is in the transition regime. The spatial region $[-1.5,1.5] \times[-1.5,1.5]$ and the velocity region $[-8,8] \times[-8,8]$ are uniformly discretized into $64 \times 64$ and $32 \times 32$ meshes, respectively. The time step is $\Delta t=0.002$ and the maximum CFL number is 0.875 .

collisionless flows. This unique feature cannot be implemented by the probabilistic methods.

In practice, since $n(x, y)$ is very small near the boundary, numerical error emerges when calculating the macroscopic velocity. Hence it is possible to get negative temperature, which is not physical. To tackle this problem, the collision term in Eq. (5) is neglected near the spatial boundary. This is justified by the fact that far from the trap center the gas is in the collisionless limit so the collision term is negligible. Another point one should pay attention to is that, the maximum Courant-FriedrichsLewy (CFL) number $\Delta t \cdot \max \left\{\left|v_{x}\right| / \Delta x+\left|v_{y}\right| / \Delta y+\right.$ $\left.\left|a_{x}\right| / \Delta v_{x}+\left|a_{y}\right| / \Delta v_{y}\right\}$ with $\Delta x, \Delta y$ the spatial steps and $\Delta v_{x}, \Delta v_{y}$ the velocity steps, must be smaller than 1 .

Numerical results for the harmonic potential. To validate the numerical scheme, we first simulate the sloshing and breathing modes in the radial isotropic harmonic trap with $\omega_{x}, \omega_{y}=\omega_{0}$. The local relaxation time is given by Eq. (5). However, the use of Eq. (6) will give the same result because the cloud is nearly isothermal, i.e., after normalization, only $n(x, y)$ affects $\tau(x, y)$. The numerical results in Fig. 1 show that, as expected, the sloshing and breathing modes oscillate with the frequency $\omega_{0}$ and $2 \omega_{0}$, respectively $[5,10]$. Note that the numerical simulations were carried out in the transition regime, where damped modes are suppressed rapidly. The two perfectly undamped modes prove the accuracy of the numerical scheme.

Now we simulate the radial quadrupole mode. Analytically, replacing the local relaxation time by the average relaxation time $\widetilde{\tau}=2 \sqrt{2} \tau(0,0)$ and applying the method of moments up to the second-order, one find that the 


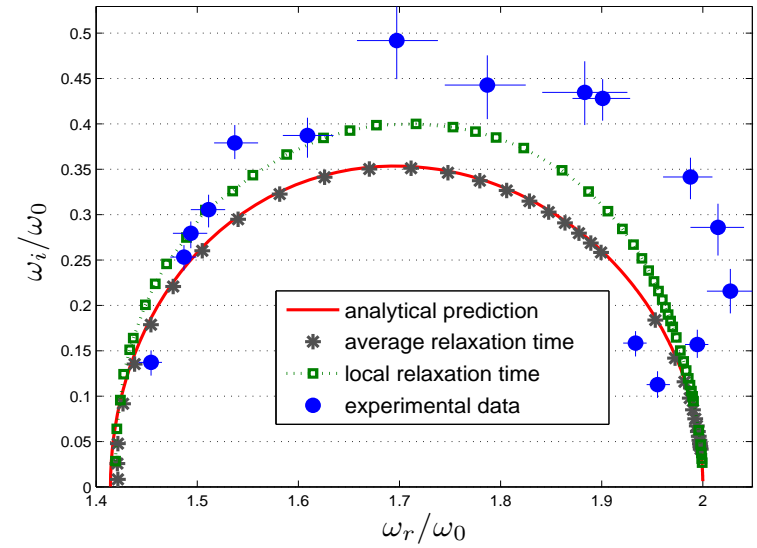

FIG. 2: (Color online) Damping rate $\omega_{i}$ versus collective frequency $\omega_{r}$ of the radial quadrupole mode. The simulation results are obtained by fitting the quadrupole moment $Q=$ $\left\langle x^{2}-y^{2}\right\rangle$ through the equation $Q(t)=A \exp \left(-\omega_{i} t\right) \sin \left(\omega_{r} t+\right.$ $\phi)+B \exp (-C t)$. The quadrupole mode is excited by initial distribution function $\exp \left\{-\left[\omega_{0}^{2}\left(x^{2}+y^{2}\right)+\left(v_{x}-0.8 x\right)^{2}+\right.\right.$ $\left.\left.\left(v_{y}+0.8 y\right)^{2}\right] / 2\right\} / 2 \pi$. The value of cross section $\sigma$ is varied to change the system from the hydrodynamic limit to the collisionless limit. Other parameters are the same as those in Fig. 1. For the experimental data, $\omega_{0}$ represents the frequency of the sloshing mode when the gas is trapped in the Gaussian potential [9].

mode frequency $\omega_{r}$ and damping rate $\omega_{i}$ satisfy the following equation:

$$
\omega^{2}-2 \omega_{0}^{2}-i \omega \widetilde{\tau}\left(\omega^{2}-4 \omega_{0}^{2}\right)=0
$$

where $\omega=\omega_{r}-i \omega_{i}[6,20]$. In the hydrodynamic regime $(|\omega \widetilde{\tau}| \ll 1)$, the mode frequency is $\sqrt{2} \omega_{0}$, while in the collisionless regime $(|\omega \widetilde{\tau}| \gg 1)$, the mode frequency is $2 \omega_{0}$.

One would expect that, if using the average relaxation time in the numerical simulation, the relation between the mode frequency and damping should, to some extent, coincide with the analytical one. This is confirmed in Fig. 2: both results (solid line and stats) match extremely well in the collisionless and transition regimes, while a small discrepancy emerges in the hydrodynamic limit. This may be due to that the second-order trial function is oversimplified [6]. Overall, this match also shows the validation of the present numerical scheme.

When the local relaxation time is used, the relation between the mode frequency and damping (squares) is very close to the analytical prediction in the collisionless and hydrodynamic limits. However, in the transition regime, the analytical damping rates are significantly underpredicted, while the numerical results are close to the experimental data. In addition, neglecting high-order moments may affect analytical results: for the quadrupole mode in the three-dimensional harmonic potential, it was found that the fourth-order moments can give better results [10].

Finally, we simulate the scissors mode in the elliptical harmonic potential. The method of moments predicts

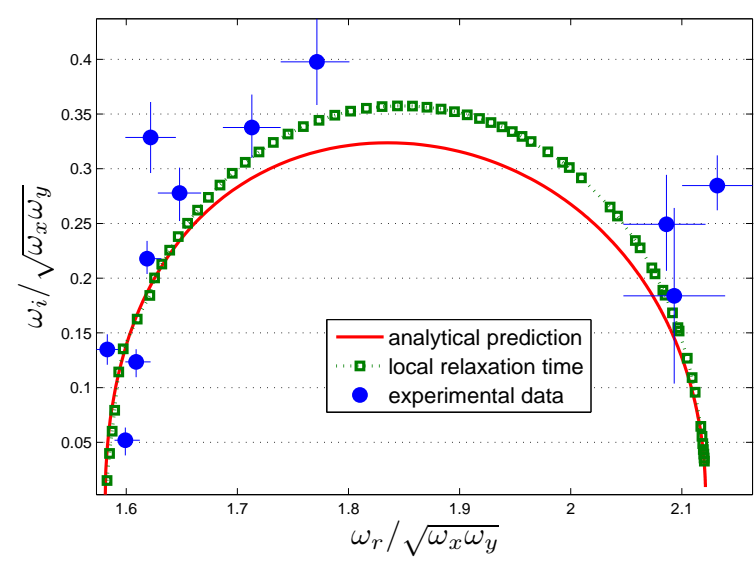

FIG. 3: (Color online) Damping rate $\omega_{i}$ versus collective frequency $\omega_{r}$ of the radial scissors mode. The simulation results are obtained by fitting the cloud angle $\theta(t)=$ $90 \operatorname{atan}\left[\langle x y\rangle /\left\langle x^{2}-y^{2}\right\rangle\right] / \pi$ to a sum of two damped sine functions each with their own free parameters. Only the higher frequency and the corresponding damping rate is plotted. The scissors mode is excited by sudden rotation of the trap angle $\theta(0)$ by $5^{\circ}$. The trap frequencies are $\omega_{x}=4$ and $\omega_{y}=2$. The time step is $\Delta t=0.0025$ and the maximum CFL number is 0.82 . Other parameters are the same as those in Fig. 1, except the spatial region in the $y$ direction is now $[-3,3]$.

the following relation between the mode frequency and damping rate:

$$
i \omega\left(\omega^{2}-\omega_{h}^{2}\right)+\widetilde{\tau}\left(\omega^{2}-\omega_{c 1}^{2}\right)\left(\omega^{2}-\omega_{c 2}^{2}\right)=0,
$$

where $\omega_{h}=\left(\omega_{x}^{2}+\omega_{y}^{2}\right)^{1 / 2}$ is the frequency in the hydrodynamic limit, $\omega_{c 1}=\omega_{x}+\omega_{y}$ and $\omega_{c 2}=\left|\omega_{x}-\omega_{y}\right|$ are the frequencies at the collisionless limit [13]. From Fig. 3 we again see that, the numerical results agree with the analytical solutions in the hydrodynamic and collisionless limits, while the analytical damping rates are smaller than the numerical ones in the transition regime. Compared with the experimental data (open circles in Fig. 2 of Ref. [11]; the last three data are magnified by a factor of 1.08 because for the harmonic potential the mode frequency in the collisionless regime should be $1245 \times 2 \pi \mathrm{Hz}$ instead of $1150 \times 2 \pi \mathrm{Hz}$ ), we find that the numerical results fit with the experiment data better than the analytical solutions.

Numerical results for the Gaussian potential. Instead of the harmonic potential, the gases are trapped in the Gaussian potential at higher temperatures. The moment method fails to provide analytical solution for the Gaussian potential, so we have to rely on numerical simulations. To calculate the collective frequency and damping of the radial quadrupole mode, the following experimental data are used [9]: $V_{0}=50 k_{B}(\mu K)$, $a, b=32.8 \mu \mathrm{m}$, with the corresponding trap frequency $\omega_{x}, \omega_{y}=1800 \times 2 \pi(\mathrm{Hz})$. The trap frequency in the $z$ direction is $\omega_{z}=32 \times 2 \pi \mathrm{Hz}$, and the total number of atoms is $N=6 \times 10^{5}$. In numerical simulations, the time, spatial coordinates, velocity, and temperature are 

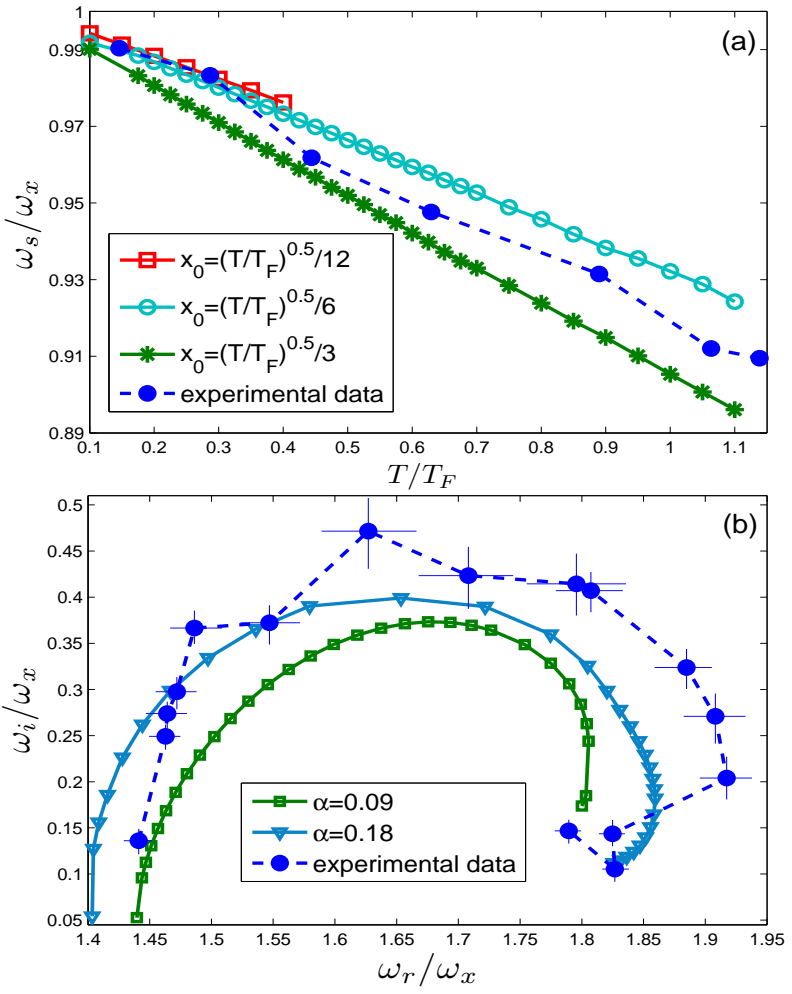

FIG. 4: (Color online) (a) The sloshing mode frequency versus the temperature in the Gaussian potential. (b) Damping rate $\omega_{i}$ versus collective frequency $\omega_{r}$ of the radial quadrupole mode. The spatial region $[-\sqrt{\widetilde{T}}, \sqrt{\widetilde{T}}] \times[-\sqrt{\widetilde{T}}, \sqrt{\widetilde{T}}]$ and the velocity region $[-8 \sqrt{\widetilde{T}}, 8 \sqrt{\widetilde{T}}] \times[-8 \sqrt{\widetilde{T}}, 8 \sqrt{\widetilde{T}}]$ are uniformly discretized into $64 \times 64$ and $32 \times 32$ meshes, respectively. The time step is $\Delta t=0.0013$.

respectively normalized by $a \sqrt{m / k_{B} T_{F}}, a, \sqrt{k_{B} T_{F} / m}$, and the Fermi temperature $T_{F}=2.73 \mu \mathrm{K}$. The distribution function is also normalized by the particle den- sity at the trap center. Therefore, the normalized accelerations in the $x$ and $y$ directions are respectively $-36.6 x \exp \left(-x^{2}-y^{2}\right)$ and $-36.6 y \exp \left(-x^{2}-y^{2}\right)$, and at the unitarity limit, the normalized local relaxation time is $\tau(x, y)=0.09\left(T / T_{F}\right)^{2} / n(x, y)$.

Figure 4(a) shows that the normalized sloshing mode frequency decreases as the temperature increases, which coincides with the experimental observations. We also find that the frequency decreases as the initial cloud center $x_{0}$ increases. Note that the sloshing mode is excited by shifting the Gaussian potential by $x_{0}$ in the $x$ direction.

Figure 4(b) shows the relation between the mode frequency and damping rate, where the local relaxation time is $\tau(x, y)=\alpha \widetilde{T}^{2} / n(x, y)$, the initial distribution function is $f=\exp \left\{-18.3\left[1-e^{-x^{2} / 1.05^{2}-1.05^{2} y^{2}}\right] / \widetilde{T}\right\} \exp \left[-\left(v_{x}^{2}+\right.\right.$ $\left.\left.v_{y}^{2}\right) / 2 \widetilde{T}\right] / 2 \pi \widetilde{T}$, and $\widetilde{T}=T / T_{F}$. When $\alpha=0.09$, the trend agrees with the experimental finding. When $\alpha=0.18$, the numerical results agree reasonably well with the experiment data. This indicates that the difference between the numerical and experiment results may be a consequence of the approximation of the relaxation rate or the neglected interaction term in Eq. (3), rather than the anharmonic effect [9].

Conclusions. To improve accuracy and overcome the limitation of the moment method, we have demonstrated a computationally efficient numerical scheme to solve the Boltzmann model equation. The extracted mode frequency and damping of the radial quadrupole and scissors modes provide better agreement with the experimental date than the analytical solutions obtained from the method of moments. The advantage of this numerical approach is that it can deal with the harmonic and Gaussian potentials, and other forms of the potential, including the mean-field and other self-energy terms, which will help us to understand the properties of strongly interacting particles.
[1] F. Dalfovo, S. Giorgini, L. P. Pitaevskii, and S. Stringari, Rev. Mod. Phys. 71, 463 (1999).

[2] S. Giorgini, L. P. Pitaevskii, and S. Stringari, Rev. Mod. Phys. 80, 1215 (2008).

[3] C. Cao, E. Elliott, J. Joseph, H. Hu, J. Petricka, T. Schäfer, and J. E. Thomas, Science 331, 58 (2011).

[4] E. A. Uehling and G. E. Uhlenbeck, Phys. Rev. 43, 552 (1933).

[5] D. Guéry-Odelin et al., Phys. Rev. A 60, 4851 (1999).

[6] U. Al Khawaja, C. J. Pethick, and H. Smith, J. Low Temp. Phys, 118, 127 (2000).

[7] P. Massignan, G. M. Bruun, and H. Smith, Phys. Rev. A 71, 033607 (2005).

[8] G. M. Bruun and H. Smith, Phys. Rev. A 76, 045602 (2007).

[9] S. Riedl et al., Phys. Rev. A 78, 053609 (2008).

[10] T. Lepers et al., Phys. Rev. A 82, 023609 (2010).

[11] M. J. Wright et al., Phys. Rev. Lett. 99, 150403 (2007).
[12] A. Altmeyer et al., Phys. Rev. A 76, 033610 (2007).

[13] G. M. Bruun and H. Smith, Phys. Rev. A 76, 045602 (2007).

[14] G. M. Kavoulakis, C. J. Pethick, and H. Smith, Phys. Rev. A 57, 2938 (1998).

[15] M. Braby, J. Chao, and T. Schäfer, Phys. Rev. A 82, 033619 (2010).

[16] E. Zaremba, T. Nikuni, and A. Griffin, J. Low Temp. Phys. 116, 277 (1999)

[17] S. Watabe, A. Osawa, and T. Nikuni, J. Low Temp. Phys. 158, 773 (2010).

[18] F. Filbet and S. Jin, J. Sci. Comput. 46, 204 (2011).

[19] S. Chapman and T. G. Cowling, The Mathematical Theory of Non-Uniform Gases (Cambridge University Press, Cambridge, 1970).

[20] Ch. Buggle et al., Phys. Rev. A 72, 043610 (2005). 